\title{
Performance Enhancement for Tsunami Wave Simulation using Hexagonal Cellular Automata
}

\author{
E.Syed Mohamed ${ }^{1}$ \\ Computer Science and Engineering Department, \\ B.S.Abdur Rahman University, Vandalur \\ Chennai 600 048, INDIA
}

\author{
S.Rajasekaran ${ }^{2}$ \\ Mathematics Department, \\ B.S.Abdur Rahman University, Vandalur \\ Chennai 600 048, INDIA
}

\begin{abstract}
Tsunamis are considered the most devastating natural hazard on costal environments ever known. Early tsunami wave detection, both quick and appropriate intervention is of vital importance for minimization tsunami damage. Simulation of tsunami wave spread remains a daunting task due to factors such as complex wave behavior, dynamical wave condition and large spatial data that needs to be modelled. In this paper tsunami wave models have been widely studied using cellular automata(CA) that has special features for simulating complex phenomena. The influential factors for tsunami wave are divided into two categories and our models are applied to eight basic cases depending on weather, topography and wave conditions for different rates of spread. The algorithm is efficient and easily implemented, allowing less computational time and cost. Experimental results of this model prove its value in tsunami wave spread as real time.
\end{abstract}

\section{Key words}

Tsunami wave, Simulation, Homogeneous, Nonhomogeneous, Cellular automata, Discrete time step, Primary wave front, Secondary wave front

\section{INTRODUCTION}

A significant deformation of an ocean floor caused by an underwater earthquake, landslide or volcanic eruption produces a tsunami wave on the ocean surface. Once the tsunami is generated, it propagates into more and more shallow ocean, and its propagation is influenced by the depth of the ocean. Since the energy of the tsunami is dissipated by the death of the ocean [1]. It is found that the propagation of the tsunamis depends on the relative magnitude of the given speed of the running speed of the running ocean and the wave speed of Shallow Ocean. Nowadays, assessing hazard conditions related to complex natural phenomena increasingly takes advantage of computer-assisted analyses and simulations. To study the tsunami wave, tsunamis are divided in to a matrix of identical square cells, with side length $\mathrm{L}$, and it is represented by a CA.

The remainder of the section is organized as follows: Section2 describes the related works available in the literature. In section3, the assumptions are used in cellular automata model. Section 4 describes multi models of cellular automata for tsunami wave spread. In section 5 the implementation of homogeneous hexagonal cellular automata models are proposed and several tests for the new models are checked and their simulation are shown. In Section 6, the implementation of non-homogeneous Hexagonal cellular automata model is proposed and several tests for the new models are checked and their simulations are shown. In Section 7, comparison with two-dimensional square cellular automata graph results shown and finally, the conclusions are presented in the paper

\section{RELATED WORKS IN THE LITERATURE}

In the open ocean a tsunami is less than a few feet high at the surface, but its wave height increases rapidly in shallow water. But, as the tsunami reaches shallower coastal waters, wave height can increase rapidly [2] [3]

A trans-oceanic tsunami is one that propagates throughout the ocean in which it is generated and could cause loss of life and damage even far away from the epicenter area. Second, an ocean-wide tsunami is one which propagates throughout the ocean in which it is generated, but the loss of life and damage are mostly confined to the epicenter area [4]

For a tsunami generated by pure thrust faulting, only the primary wave fronts would be evident: one moving toward the deep ocean and one moving toward the local shoreline

In addition, there is a secondary wave front propagating to the northeast that is a continuation of the shoreward primary wave front. Both of the secondary wave fronts initially travel parallel to shoreline, but their paths of travel curve (refract) toward shore.

Physics tells us that when the energy in a system remains constant, but velocity decreases, the mass in the system must increase. A slower moving tsunami is a physically higher tsunami. The waves scrunch together like the ribs of an accordion and heave upward. [5]

In this work, a new cellular automaton model based on the transfer of fractional traversed area for tsunami propagation in real time simulation and visualization is proposed [6].

The long term goal of this work is to develop a simulation tool that can be used in real-time or as-fast-as-can tactical decision making to aid in tsunami wave spread and suppression.

This simulation model was developed using Java. It is used for finding the rate of spread of the tsunami wave under two types of ocean, eight topological and wave conditions. 


\section{ASSUMPTIONS USED IN CELLULAR AUTOMATA MODEL}

A cellular automaton is an array of 'cells' that interact with their neighbours. These arrays can take on any number of dimensions, starting from a one dimensional string of cells. Each cell has its own state that can be a variable, property or other information.

At the beginning of the simulation, cell states are initialized by means of input matrices. Moore's model parameters have also to be assigned in this phase, by taking into consideration their physical/empirical meaning. By simultaneously applying the transition function to all the cells, at discrete steps, states are changed and the evolution of the phenomenon can be simulated

A tsunami wave model represented as a two-dimensional cellspace composed of cells of dimensions $\ell \times b$, where $\ell$ and $b$ are the length and breadth of the cell, respectively. For each cell , 8 fixed major spread directions (propagation lines) $\mathrm{N}$, NE, E, SE, S, SW, W, and NW are defined as shown in Fig.1.

This allows for the computation of wave spread in only the specified major directions instead of all directions and thus, significantly reduces wave spread computation time.

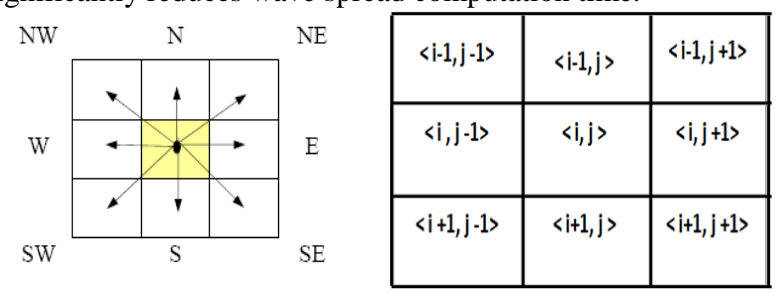

Fig.1 Potential neighbour cells to spread by wave from center cell directional movements

\subsection{Hexagonal Cellular Automata and Its Features}

The two-dimensional cellular automaton [7] does not have accuracy in the shape of the output obtained. Further the rate of spread is high, which decreases the efficiency [8]. Hence the hexagonal cellular automata are used, in which the spread is not linear, the rate of spread is low and the shape of the output is very similar to that obtained in real tsunamis.

Let us focus for simplicity on a single CA cell (individuated as the "central" cell) of the two-dimensional space: it is considered limited to the universe of its neighbourhood, which consists of $\mathrm{m}$ cells (the central cell and its adjacent cells). Indexes are utilised to individuate the central cell (0) and the adjacent ones $(1,2 \ldots m-1)$, respectively. Twodimensional cellular automata $(\mathrm{CA})$ are discrete dynamical systems formed by a finite number of identical objects called cells, which are arranged uniformly in a two dimensional space [9]. They are endowed with a state that changes at every discrete step of time according to a deterministic rule. More precisely, a CA can be defined as a 4-tuplet $U=(R, N, Q, k)$ where $\mathrm{R}$ is the cellular space formed by a two-dimensional array of $q x b$ cells: $\quad\{(x, y), 1 \leq x \leq q, 1 \leq y \leq b\}, \quad$ such that each of which can assume a state. In the bi-dimensional case, the cells are usually represented as identical square areas (Fig.2 ), but in this work, the cells will be represented by means of regular hexagonal areas (Fig. 2), making a tessellation of the plane. This new representation allows us to obtain a more realistic simulation [1].

\begin{tabular}{|l|l|l|l|l}
\hline$(1,1)$ & $(1,2)$ & $(1,3)$ & $(1,4)$ & \\
\hline$(2,1)$ & $(2,2)$ & $(2,3)$ & $(2,4)$ & \\
\hline$(3,1)$ & $(3,2)$ & $(3,3)$ & $(3,4)$ & \\
\hline & & & &
\end{tabular}

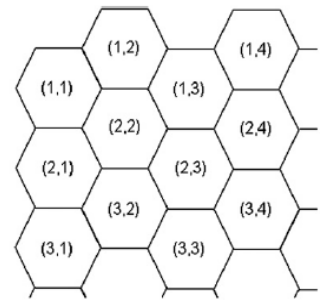

Fig.2. Square cellular space and Hexagonal cellular space

\subsection{The Cellular Automata Based Model For Tsunami Wave Spreading}

In this section the model for predicting tsunami wave spreading based on two-dimensional linear cellular automata with hexagonal cellular space is proposed. In this model ocean area can be interpreted as the hexagonal cellular space by simple dividing it into a two-dimensional array of identical hexagonal areas of side length $L$. Obviously, each one of these areas stands for a cell of the CA.
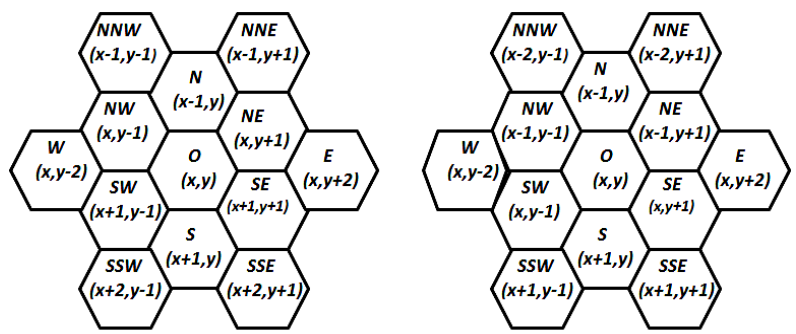

Fig 3. Even neighbour cells

Fig.4. Odd neighbour cells

The state of a cell $(\mathrm{x}, y)$ at a time $t$, is defined as follows:

$N_{x y}^{(t)}=$ traversed area of $(\mathrm{x}, y)$ at a time $t /$ total area of $(x, y)$ where as a simple calculus shows, the total area of the hexagonal cell $\quad(x, y)$ is $3 \sqrt{ } 3 L^{2} / 2$.If $N_{x y}^{(t)}=0$, then the cell $(\mathrm{x}, y)$ is said to be untraversed at time $t$; if $0<N_{x y}^{(t)}<1$, then the cell $(\mathrm{x}, y)$ is partially traversed out at time $t$, and finally if $N_{x y}^{(t)}=1$, the cell is said to be completely traversed out at time $t$. Observed that the values $N_{x y}^{(t)}$ may else be greater than 1 . In this case, the state of the cell $(x, y)$ at time $t$ is taken to be equal to 1 .

The dynamics of such automata basically supposes that the state of a cell $(x, y)$ at time $(t+1)$ linearly depends on the states of its neighbour cells at time $t$; specifically one has 


$$
\begin{aligned}
N_{x y}^{(t+1)}= & f\left(N_{x y}^{(t)}+\sum_{(\lambda, \delta) \in Q_{n}} \mu_{\lambda \delta}^{(x, y)} N_{x+\lambda, y+\delta}^{(t)}+\right. \\
& \left.\sum_{(\lambda, \delta) \in Q_{d}} \mu_{\lambda \delta}^{(x, y)} N_{x+\lambda, y+\delta}^{(t)}\right)
\end{aligned}
$$

where $\mu_{\lambda \delta}^{(x, y)} \in \mathbb{Z}$ are parameters involving some physical magnitudes of the cells, and the discretization function $f$ is given by

$\mathrm{f:}[0,1] \rightarrow N$

$a \mapsto f(a)=\frac{\left[10_{a}\right]}{10}$,

where $[c]$ stands for the closest integer to $\mathrm{c}$.

As it is mentioned above, each cell $(x, y)$, represents a small hexagonal area of the ocean. Then, it is endowed with three parameters: the rate of wave $\operatorname{spread} R A_{(x, y)}$, the wave speed $W A_{(x, y))}$, and the depth $D E_{(x, y)}$ of the cell. Consequently, the expression of the parameter $\mu_{\lambda \delta}^{(x, y)}$ is as follows:

$\mu_{\lambda \delta}^{(x, y)}=\omega a_{\lambda \delta}^{(x, y)} \cdot d e_{\lambda \delta}^{(x, y)} \cdot r a_{\lambda \delta}^{(x, y)}$ Where $W A_{(x, y)}$ stands for the wave influence of the neighbor cell $(x+\lambda, y+\delta)$ on $(\mathrm{x}, \mathrm{y})$, such that $W A_{(a, b)}=\left\{\omega a_{\lambda \delta}^{(x, y)},(\lambda, \delta) \in Q\right\} ; d e_{\lambda \delta}^{(x, y)}$ represents the height influence and, as is shown below, it is a function of $D E_{(x, y)}-D E_{(x-\lambda, y+\delta)}$ where $D E_{(x, y))}$ is the height in the central point of the hexagonal area which is represented by the cell $(x, y)$. It is supposed that this height is the same in every point of such cell. Finally, $r a_{\lambda \delta}^{(x, y)}$ is a parameter which stands for the influence of the different rates of tsunami wave spread.

\subsection{The Size of Discrete Time Step}

Since cellular automata evolve in discrete time steps, it is a basic point to decide the size of such step of time $\tilde{t}$. In the proposed model, this step is equal to the time needed for a one and only near neighbor cell to be traversed.

The rate of tsunami wave spread of the cell $(x, y)$, $R A_{(x, y)}$, determines the time needed for this cell to be completely traversed out and depends on the physical composition of the cell [10]. Note that if the cell $(x, y)$ stands for an untraversed hexagonal area, then $R A_{(x, y)}=0$ and $N_{x y}^{(t)}$ $=0$ for every $\mathrm{t}$.

The importance of this parameter lies in the setting-up of the size of the time step $\tilde{t}$. Suppose that the ocean area modeled is homogeneous, i.e. the value of the rate of wave spread is the same for all cells : $\left\{R A_{(x, y)}, 1 \leq x \leq q, 1 \leq y \leq b\right\}$

Then, it is easy to check that if the only traversed cell at time $\mathrm{t}$ in the neighbourhood of $\mathrm{O}$ is for example $\mathrm{N}$, then the time needed for $\mathrm{O}$ to be completely traversed out is $\quad \tilde{t}=\sqrt{3} \frac{L}{R}$

Consequently, if all cells in the neighbourhood of $O=(x, y)$ are untraversed at time t except only one adjacent cell, which is completely traversed out, then at time $\mathrm{t}+1$, the cell $(x, y)$ is completely traversed: $N_{x y}^{(t+1)}=1$

However, since almost all real oceans are nonhomogeneous, the step size is taken to be the time needed for the cells with the larger rate spread to be completely traversed out,that is

$$
\tilde{t}=\sqrt{3} \frac{L}{R}
$$

where $\mathrm{RA}=\max \left\{R A_{(x, y)}, 1 \leq x \leq q, 1 \leq y \leq b\right\}$

It is followed that if the only completely traversed out neighbour cell at time $t$, is a distant neighbour cell of $O=(x, y)$, say NNE, then $N_{x y}^{(t+1)}=\gamma<1$. That value is calculated as follows. In a step time $\tilde{t}$, the near neighbor cells of the cell NNE, N and NE, and a little portion (a circular sector) of the cell $\mathrm{O}$ will be traversed out. Specifically, as the distance covered by the tsunami wave spread in $t$ with speed $\mathrm{R}$ is $\sqrt{3} \mathrm{~L}$ then the radius of the circular sector of $O$ traversed out is $\sqrt{3} L-L$

As a consequence, the traversed out area of the cell $O$ will be

$$
\frac{\pi(\sqrt{3}-1)^{2} L^{2} \frac{2 \pi}{3}}{2 \pi}=\frac{4-2 \sqrt{3}}{3} \pi L^{2}
$$

So, if all neighbour cells of $(a, b)$ are un traversed at time t, except a distant neighbour which is fully traversed out, then

$N_{x y}^{(t+1)}=\gamma=\frac{\frac{4-2 \sqrt{3}}{3} \pi L^{2}}{\frac{3 \sqrt{3}}{2} L^{2}}=\frac{8 \sqrt{3}-12}{27} \pi \approx 0.216$

\subsection{The Influence of Tsunami Wave}

Another factor to be incorporated to the model is the wave speed and direction, due to their important influence to the tsunami wave spreading [7]. As was stated above, the effect of the wave on the cell $\mathrm{O}$ is given by the set

$W A_{(x, y)}=\left\{\omega a_{\lambda \delta}^{(x, y)},(\lambda, \delta) \in Q\right\} ;$

where $\omega a_{\lambda \delta}^{(x, y)}>0$, in such a way that if no wave is traversed on $O=(x, y)$ then $\omega a_{\lambda \delta}^{(x, y)}=1$ for every $(\lambda, \delta) \in V$; if the wave is traversed from North to South, then the coefficients $\omega a_{N W}^{O}, \omega a_{N N W}^{O}, \omega a_{N}^{O}, \omega a_{N N E}^{O}, \omega a_{N E}^{O}$ must be larger than the rest of coefficients, and so on. The value of such coefficients stand for the magnitude of the wave.

\subsection{The Influence of Topography}

The height differences between various points in a ocean also affects to the wave spreading. As is well-known, the waves show a higher rate of spread when they descend, whereas waves show a smaller rate of spread when they ascend.The height influence of a near neighbour cell $(x+\lambda, y+\delta)$, on a cell $O=(x, y)$ is given by $d e_{\lambda \delta}^{(x, y)}$, which depends on the difference of height between each pair of cells considered, that is,

$$
d e_{\lambda \delta}^{(x, y)}=\varnothing\left(H A_{(x, y)}-H A_{(\mathrm{x}+\lambda, y+\delta)}\right)
$$

The function $=\varnothing(a)$, where $\mathrm{x}$ stands for the height difference, must be determined according to the characteristic of the tsunami, and, also, it has to satisfy the following conditions:

$$
\begin{aligned}
& \text { If } a>0 \text {, then } \emptyset(a)<1 \\
& \text { If } a=0 \text {, then } \emptyset(a)=1 \\
& \text { If } a<0 \text {, then } \emptyset(a)>1
\end{aligned}
$$

Note that the first condition establishes that if 
$D E<D E_{(\mathrm{x}+\lambda, y+\delta)}$, the tsunami wave increases its rate of spread; the second condition states that when $D E_{(x, y)}=D E_{(\mathrm{x}+\lambda, y+\delta)}$, the topography does not affect to the tsunami wave spread; and the third condition establishes that if $D E_{(x, y)}>D E_{(x+\lambda, y+\delta)}$ the tsunami wave restrains its spreading. Moreover, the height influence of a distant neighbour cell is affected by the influence of its associated near neighbour cells.

For example, if the distant neighbour cell of $\mathrm{O}$ is NNE, then:

$d e_{N N E}^{O}=\frac{1}{4}\left[\varnothing\left(D E_{O}-D E_{N}\right)+\emptyset\left(D E_{N}-D E_{N N E}\right)+\right.$

$\varnothing D E O-D E N E+\varnothing D E N E-D E N N E$ and so on

Note that if the tsunami is horizontal wave motion (all cells have the same height), then $\varnothing(a) \leq 1$ and consequently $d e_{\lambda \delta}^{(x, y)}=1$ for every cell $(\mathrm{x}, \mathrm{y})$ and $\operatorname{any}(\lambda, \delta) \in Q$

\subsection{The Rate of Wave Spread}

Let us consider a non-homogeneous tsunami and set $\mathrm{R}$ the maximum rate of wave spread. Let $O=(x, y)$ be a cell with $R A_{O} \leq R \mathrm{~A}$. The purpose of this section is to determine the value of the parameter, $q_{\lambda \delta}^{(x, y)}$, which stands for the influence of the different rates of wave spread of the neighbor cells.If all neighbour cells are untraversed at time t except only one near neighbour cell of $\mathrm{O}$, say for example $\mathrm{N}$, then after a time step, $\tilde{t}$ the space traversed by the wave spread is given by :

$$
q=R A_{0} \tilde{t}=\sqrt{3 \frac{R A_{O}}{R A} L}
$$

and consequently, there are two cases to be considered: When $q \leq L$, and when $\mathrm{q}>\mathrm{L}$.

If $q \leq L$, then $\sqrt{3} \frac{R A_{O}}{R A} L \leq L$, and $\frac{R A_{O}}{R A} \leq \frac{\sqrt{3}}{3} \approx 0.57735$

As a consequence, the traversed out area of the cell $\mathrm{O}$ after a time step $\sim \mathrm{t}$ is given by $L_{q}+2 \frac{q^{2 \frac{\pi}{6}}}{2}=\left(\sqrt{3}+\frac{\pi}{2} \frac{R A_{O}}{R A}\right) \frac{R A_{O}}{R A} L^{2}$

Consequently,

$$
q_{N}^{O}=\frac{\left(\sqrt{3}+\frac{\pi}{2} \frac{R A_{O}}{R A}\right) \frac{R A_{O}}{R A} L^{2}}{\frac{3}{2} \sqrt{3} L^{2}}=
$$

$$
\frac{2 \sqrt{3}}{9}\left(\sqrt{3}+\frac{\pi}{2} \frac{R A_{O}}{R A}\right) \frac{R A_{O}}{R A}
$$

If $q>L$, then $L<\sqrt{3} \frac{R A_{O}}{R A} L$, and

$$
0.57735 \approx \frac{\sqrt{3}}{3}<\frac{R A_{O}}{R A} \leq 1
$$

As a simple calculus shows, the traversed out area of the cell $\mathrm{O}=(\mathrm{x}, \mathrm{y})$ after a time step $\tilde{t}$ is given by

$$
\left[1+\sin \left(\frac{\pi}{6}-\lambda\right)+\sqrt{3} \lambda \frac{R A_{O}}{R A}\right] \sqrt{3} \frac{R A_{O}}{R A} L^{2}
$$

where,

$$
\begin{gathered}
\lambda=\frac{\pi}{6}-\arccos \left(\frac{\sqrt{3}}{4} \frac{R A}{R A_{O}}+\frac{\sqrt{3}}{12} \sqrt{12-3 \frac{R A^{2}}{R A_{o}^{2}}}\right), \\
0 \leq \lambda<\frac{\pi}{6}
\end{gathered}
$$

As a consequence:

$$
\begin{aligned}
& q_{N}^{O}=\frac{\left[1+\sin \left(\frac{\pi}{6}-\lambda\right)+\sqrt{3} \lambda \frac{R A_{O}}{R A}\right] \sqrt{3} \frac{R A_{O}}{R A} L^{2}}{\frac{3}{2} \sqrt{3} L^{2}} \\
& =\frac{2}{3}\left[1+\sin \left(\frac{\pi}{6}-\lambda\right)\right] \frac{R A_{O}}{R A}+\frac{2 \sqrt{3}}{3} \lambda \frac{R A_{o}^{2}}{R A^{2}}
\end{aligned}
$$

Note that if the tsunami is homogeneous, then $\mathrm{R}_{\mathrm{ab}}=\mathrm{R}$ for every cell $(x, y)$, and

$$
\lambda=\frac{\pi}{6}-\arccos \left(\frac{\sqrt{3}}{2}\right)=0
$$

Consequently $q_{N}^{O}=1$ was expected.On the other hand, if all neighbour cells are untraversed at time $t$, except only one distant neighbour cell, say for example NNE, then after a time step $\tilde{t}$, the wave spread traverses the border between the near neighbour cells $\mathrm{N}$ and NE, and affects the main cell $O$.

The border line between $\mathrm{N}$ and $\mathrm{NE}$ is traversed in $\tilde{t}-L / \max$ $\left\{R A_{N}, R A_{N E}\right\}$ and consequently, the space traversed along the cell $\mathrm{O}$ is:

$$
R A_{O}\left(\tilde{t}-\tilde{t}_{O}\right)=\left(\frac{\sqrt{3}}{R A}-\frac{1}{\max \left\{R A_{N}, R A_{N N E}\right\}}\right) R A_{O} L
$$

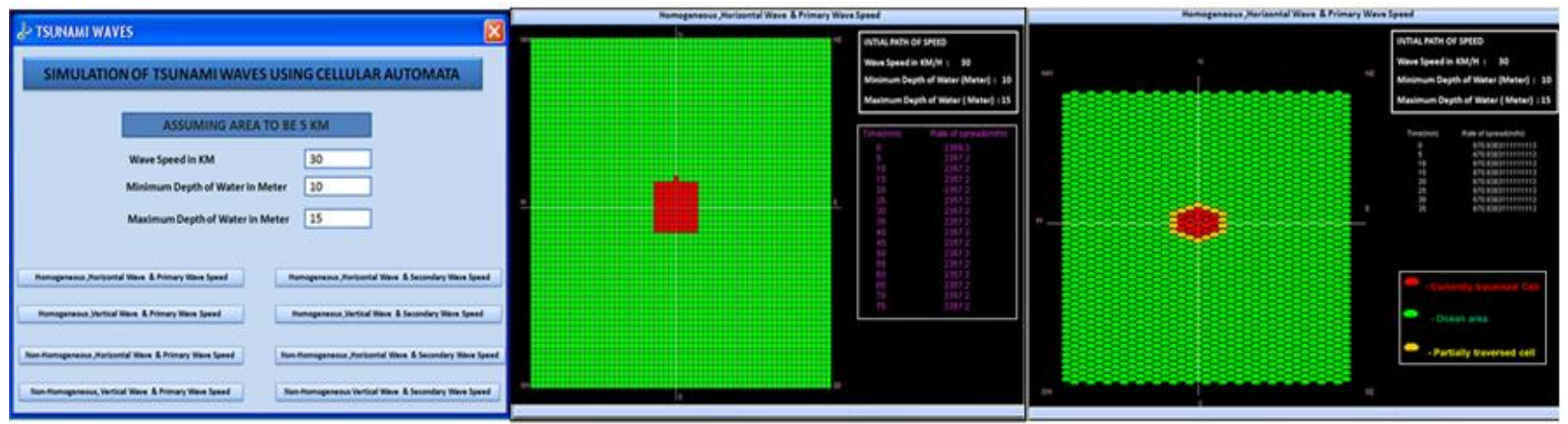

Fig.5. Comparison of two dimensional and hexagonal cellular automata simulations result for Tsunami wave propagation with different topographic conditions 
As a consequence, the traversed out area of $O$ after a time step $\tilde{t}$ is:

$$
\begin{aligned}
& \frac{\pi\left(\frac{\sqrt{3}}{R A}-\frac{1}{\max \left\{R A_{N}, R A_{N N E}\right\}}\right)^{2} R A_{O}{ }^{2} L^{2} \frac{2 \pi}{3}}{\frac{3}{2} \sqrt{3} L^{2}} \\
= & \frac{\pi}{3}\left(\frac{\sqrt{3}}{R A}-\frac{1}{\max \left\{R A_{N}, R A_{N N E}\right\}}\right)^{2} R A_{O}{ }^{2} L^{2}
\end{aligned}
$$

Obviously, the state of the cell $O$ is

$$
\begin{array}{r}
=\frac{\frac{\pi}{3}\left(\frac{\sqrt{3}}{R A}-\frac{1}{\max \left\{R A_{N} R A_{N N E}\right\}}\right)^{2} R A_{O}{ }^{2} L^{2}}{\frac{3}{2} \sqrt{3} L^{2}} \\
q_{N N E}^{O}=\frac{2 \pi \sqrt{3}}{27}\left(\sqrt{3} \frac{R A_{O}}{R A}-\frac{R A_{O}}{\max \left\{R A_{N}, R A_{N N E}\right.}\right)^{2}
\end{array}
$$

Note that if the tsunami is homogeneous, then

$q_{N N E}^{O}=\frac{2 \pi \sqrt{3}}{27}(\sqrt{3}-1)^{2}=\frac{8 \sqrt{3}-12}{27}=\gamma$

\section{SIMULATION RESULTS OF} TESTING OF MODULES

\subsection{Homogeneous Ocean Rate of Spread Calculation}

- Near Cells:

If $q \leq L$ then, $q_{N}^{O}=\frac{2 \sqrt{3}}{9}\left(\sqrt{3}+\frac{\pi}{2} \frac{R A_{O}}{R A}\right) \frac{R A_{O}}{R A}$

If $q>L$ then, $\left[1+\sin \left(\frac{\pi}{6}-\lambda\right)+\sqrt{3} \lambda \frac{R A_{O}}{R A}\right] \sqrt{3} \frac{R A_{O}}{R A} L^{2}$;

where, $\lambda=0$.

Distant Cells:

$$
q_{N N E}^{O}=\frac{2 \pi \sqrt{3}}{27}(\sqrt{3}-1)^{2}=\frac{8 \sqrt{3}-12}{27}=\gamma
$$

\subsection{Homogeneous Ocean, horizontal wave motion and Primary Wave Speed}

In the case of horizontal wave motion, the spread is even and with primary wave speed, the spread is even in all directions as shown in the Fig.6.

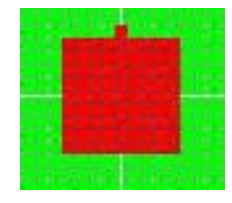

2D model

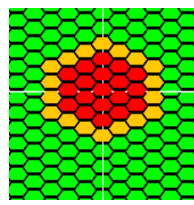

Hexagonal
Fig.6. Homogeneous Ocean, horizontal wave motion for primary wave speed and all directions.

\subsection{Homogeneous Ocean, horizontal wave motion and Secondary Wave Direction}

In this case, from the central cell, the neighbouring cells are partially traversed, and then they are completely traversed, in the specified direction. The spread is even and

with secondary wave direction, the spread is more in the specified direction as shown in Fig.7.

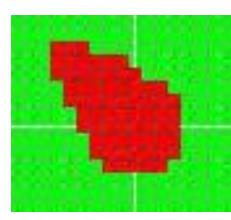

2D model

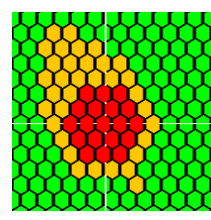

Hexagonal
Fig.7. Homogeneous Ocean, horizontal wave motion for secondary wave speed and direction in NorthWest

\subsection{Homogeneous Ocean, Vertical wave motion and Primary Wave Speed}

In this case of vertical wave motion, the spread is very fast in the trans-ocean tsunami direction, moderate on the sides if the slope and very slow down the slope. With primary wave speed, the spread is even as shown in the Fig.8.

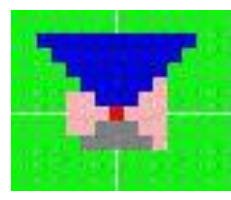

2D model

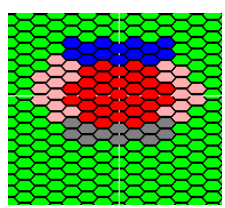

Hexagonal
Fig.8. Homogeneous Ocean, Vertical wave motion for primary wave speed

\subsection{Homogeneous Ocean, Vertical wave motion and Secondary Wave Speed}

In this case, from the central cell, the neighbouring cells are partially traversed, and then they are completely traversed, in the specified direction.

In vertical wave motion, the spread is very fast in the transocean tsunami direction, moderate in the sides of the slope and very slow down the slope. With secondary wave directions, the spread is more on the specified direction; the spread is shown in the Fig.9.

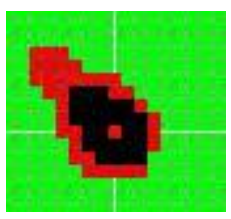

2D model

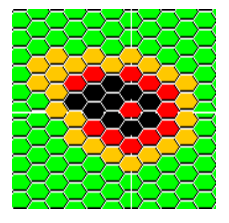

Hexagonal
Fig.9. Homogenous Ocean, Vertical wave motion with Secondary wave speed in North-West 
5 NON- HOMOGENEOUS MODULES 5.1 Non-Homogeneous Ocean, Rate of Spread Calculation

- Near Cells:

If $q \leq L$ then,

$$
q_{N}^{O}=\frac{2 \sqrt{3}}{9}\left(\sqrt{3}+\frac{\pi}{2} \frac{R A_{O}}{R A}\right) \frac{R A_{O}}{R A}
$$

If $q>L$ then,

$$
\begin{array}{r}
q_{N}^{O}=\frac{2}{3}\left[1+\sin \left(\frac{\pi}{6}-\lambda\right)\right] \frac{R A_{O}}{R A}+\frac{2 \sqrt{3}}{3} \lambda \frac{R A_{o}^{2}}{R A^{2}} ; \text { where, } \\
\lambda=\frac{\pi}{6}-\arccos \left(\frac{\sqrt{3}}{4} \frac{R A}{R A_{(x, y)}}+\frac{\sqrt{3}}{12} \sqrt{\left.12-3 \frac{R A^{2}}{R A_{(x, y)}^{2}}\right),}\right. \\
0 \leq \lambda \leq \pi / 6
\end{array}
$$

- Distant Cells:

$$
q_{N N E}^{O}=\frac{\pi}{3}\left(\frac{\sqrt{3}}{R A}-\frac{1}{\max \left\{R A_{N}, R A_{N N E}\right\}}\right)^{2} R A_{O}^{2} L^{2}
$$

\subsection{Non-Homogeneous Ocean, horizontal wave motion and Primary Wave Speed}

In the case of horizontal wave motion, the spread is even and with primary wave speed, the spread is even in all directions; which is shown in the Fig.10.

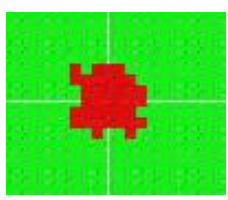

2D model

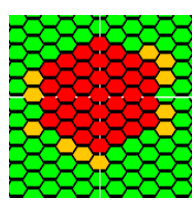

Hexagonal
Fig.10. Non-homogenous Ocean, horizontal wave motion with Primary wave

\subsection{Non -Homogeneous Tsunami, horizontal wave motion and Secondary Wave \\ Direction}

In this case of horizontal wave motion, the spread is even and with secondary wave direction, the spread is more in the specified direction, which is shown in the Fig. 11 .

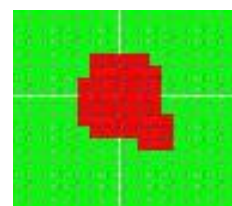

2D model

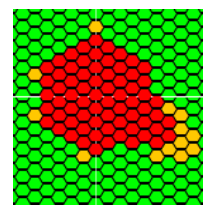

Hexagonal
Fig.11. Non-homogenous Tsunami, horizontal wave motion with Secondary wave speed in South-West

\subsection{Non -Homogeneous Ocean, horizontal wave motion and Secondary Wave Direction}

In the case of vertical wave motion, the spread is very fast in the trans-ocean tsunami direction, moderate in the sides of the slope and very slow down the slope. With primary wave speed, the spread is even; which is shown in the Fig.12.

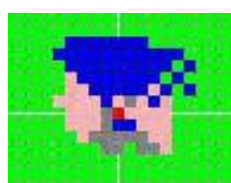

2D model

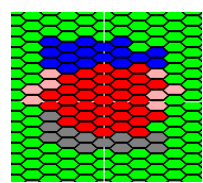

Hexagonal
Fig.12. Non-homogenous Ocean, Vertical wave motion with Primary wave speed

\subsection{Non-Homogeneous Tsunami, Vertical wave motion and Secondary Wave Direction}

In the case of vertical wave motion, the spread is very fast in the trans-ocean tsunami direction, moderate in the sides of the slope and very slow down the slope. With secondary wave direction, the spread is more in the specified direction; which is shown in the Fig.13

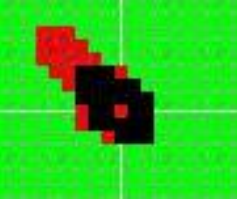

2D model

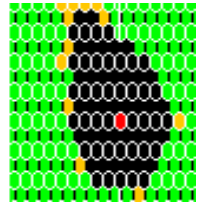

Hexagonal
Fig.13. Non-homogenous ocean, Vertical wave motion for Secondary wave speed in North-West

\section{COMPARISONS WITH TWO- DIMENSIONAL SQUARE CELLULAR AUTOMATA \\ 6.1 Rate of Spread (Homogeneous Oceans)}

Here the rate of spread of hexagonal cellular automata with that of Two-dimensional Square cellular automata for homogeneous tsunamis [10] with horizontal wave motions and primary wave are compared.

From this comparison it is found that the rate of spread is constant for both the cases, as there are same types of waves throughout the ocean. The area of a square cell is smaller than the area of a hexagonal cell, so the rate of spread in our results is less when compared to that of the Two-dimensional Square spread. 


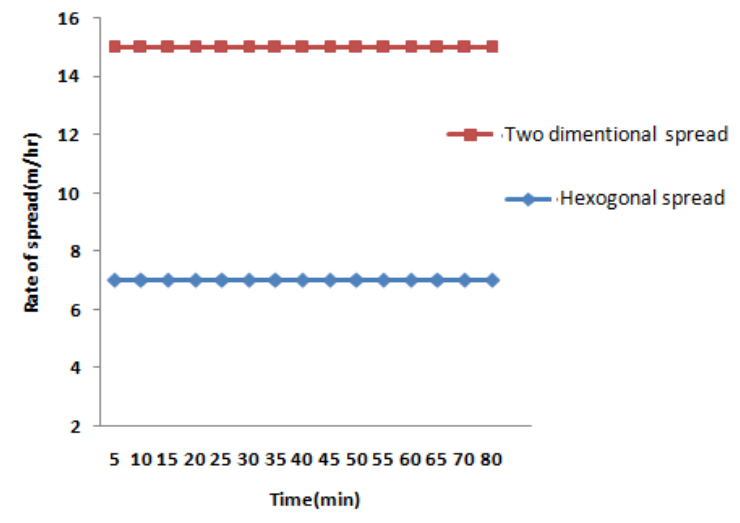

Fig.14. Comparison of rates of spread between Twodimensional Square CA and Hexagonal CA in homogeneous Ocean.

\subsection{Number of Cells Traversed (Homogeneous Oceans)}

Here the number of cells traversed in hexagonal cellular automata with that of two-dimensional square cellular automata for homogeneous oceans with Horizontal wave motion and primary wave are compared. It is seen that the number of cells traversed in our result is less when compared to that of the Two-dimensional Square model. This is because the area of a square cell is small, so the cells traversed at a particular time is more, when compared to the area of the hexagonal cells, which is large and hence the cells traversed at a particular time is less.

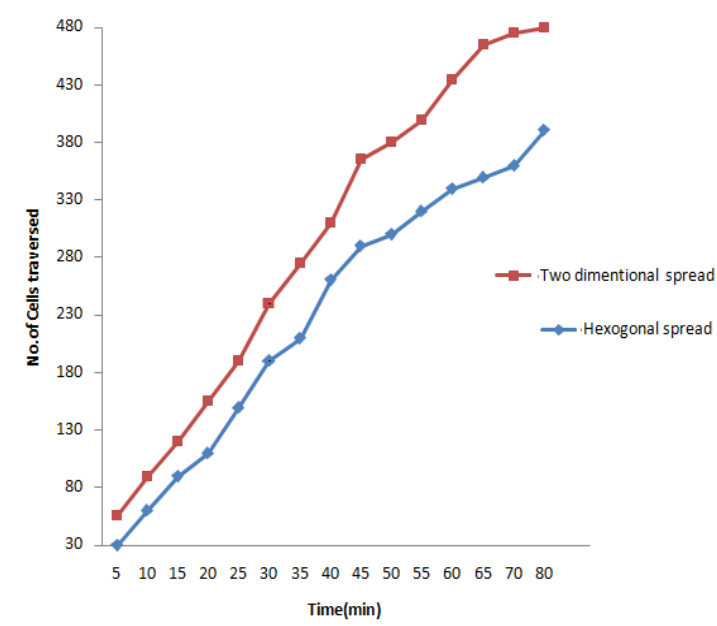

Fig.15. Comparison of no. of cells traversed between Twodimensional Square CA and Hexagonal CA in homogeneous Ocean.

\subsection{Rate of Spread (Non-Homogeneous Oceans)}

Here the rate of spread of hexagonal cellular automata with that of Two-dimensional square cellular automata for nonhomogeneous oceans with horizontal wave motions and primary wave are compared. From the graph it is seen that the rate of spread is nearly constant which is not accurate for any non-homogeneous oceans. Comparatively the rate of spread is uneven in our results, which is more compatible with the rate of spread in non-homogeneous ocean.

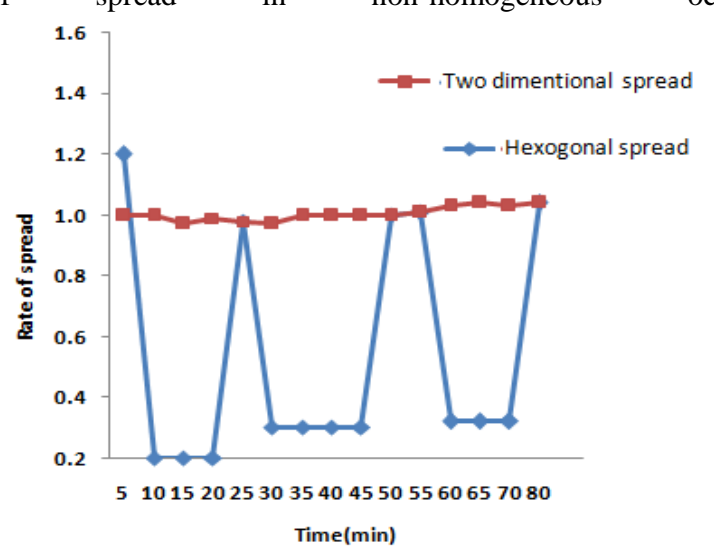

Fig.16.Comparison of rates of spread between Twodimensional Square CA and Hexagonal CA in nonhomogeneous Ocean.

\subsection{Number of Cells Traversed (Non- Homogeneous Oceans)}

Here the number of cells traversed in hexagonal cellular automata with that of two-dimensional square cellular automata for non-homogeneous oceans with horizontal wave's motion and primary waves are compared. It is seen that the number of cells traversed in two-dimensional square model is more, even though the area of the cells is less. But in this result, the area of the cells is more and the number of cells traversed is less, which is more accurate.
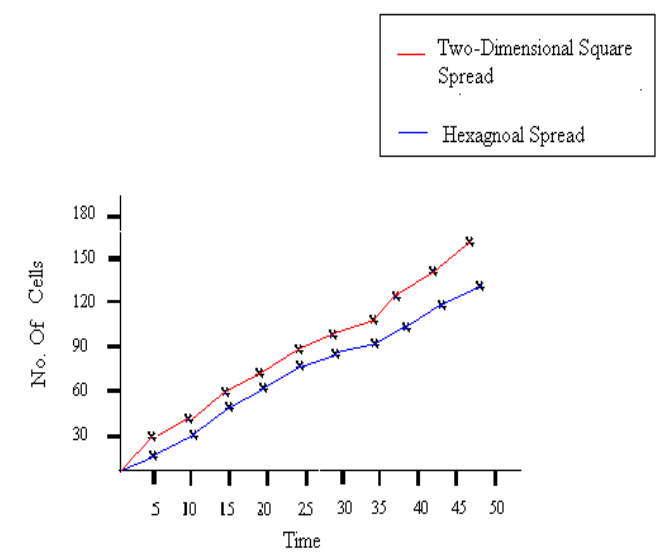

Fig.17. Comparison of no. of cells traversed between Two-dimensional square $\mathrm{CA}$ and Hexagonal $\mathrm{CA}$ in nonhomogeneous Ocean. 


\section{CONCLUSIONS}

In this work, a multi model for the prediction of tsunami wave spreading has been introduced. It is based on the basic, two dimensional, hexagonal cellular automata and incorporates weather and wave topography conditions. The states of each cell are well defined by means of transfer of fractional traversed area. Also, different rates of speed are considered. The algorithm seems to be very efficient and is easily implemented in any computer algebra system, allowing a low computational cost

The model is applied to eight basic cases depending on the weather, topography and wave speed conditions. All the graphical models obtained are found to be in agreement with wave spreading in real tsunamis.

\section{8 .REFERENCES}

[1] D.H.Zhang,T.L.Yip and C.-O.Ng,Predicting tsunami arrivals:Estimates and policy implications,Marine Policy,33(2009),643-650

[2] Yuri,I.S and Leonid B.Clubaror .,(1995),Mathematical modeling in mitigating the hazardous effects of tsunami waves in the ocean-a prior analysis and timely online forecast,Science of tsunami hazards 1391),pp27-44.
[3] "Offshore,Nearshore and Foreshore Behavior of Tsunami Waves",New Mexico heigh School Adventures in Supercomputing Challenge Final Report April24,2006.

[4] http://www.tsunami.noaa.gov/terminology.html

[5] Eric L. Geist2005, ,Local Tsunami Hazards in the Pacific Northwest from Cascadia Subduction Zone Earthquakes , U.S. Geological Survey Professional Paper 1661-B.

[6] M. H. Dao and P. Tkalich "Tsunami propagation modelling - a sensitivity study"Nat. Hazards Earth Syst. Sci., 7, 741-754, 2007,

[7] Wolfram,S.,(1983),Statistical Mechanics of Cellular Automata, Reviews of Modern Physics 55,601-644

[8] E.Syed Mohamed and S.Rajasekaran "Tsunami Wave Propagation Models based on two-dimensional Cellular Automata, International Journal of Computer Applications (0975 - 8887) Volume 57, November 2012

[9] E.Syed Mohamed and S.Rajasekaran "Tsunami Wave Propagation Models based on Hexogonal Cellular Automata(Communicated for publication)

[10] http://www.zmescience.com/science/physics/indonesia8-6-earthquake-tsunami-11042012/ 